\title{
Enhancement of $\mathrm{Pb}(\mathrm{II})$ ions adsorption onto magnesium loaded activated carbon in aqueous solution
}

Norihiko Suzuki*, Motoi Machida*,*, Yoko Fujimura**, Masami Aikawa*** and Hideki Tatsumoto*

\begin{abstract}
Magnesium loaded activated carbon was prepared to increase the adsorption capacity of lead(II) ions from aqueous solution. In case of sufficient amounts of surface acidic functional groups existing on the carbon, no significant improvement of heavy metal removal was observed even if the magnesium was loaded on it, but removing surface oxygen compounds by out-gassing in helium flow at $1173 \mathrm{~K}$ before magnesium loading, considerable enhancement of the heavy metal adsorption affinity onto the activated carbon could be observed. The amount of magnesium impregnation in the aqueous solution was also increased using the out-gassed carbon in the preparation. Any detrimental effect was not observed for aromatics adsorption by the introduction of magnesium. All adsorption equilibrium results at $293 \mathrm{~K}$ obeyed Langmuir isotherms. Heavy metal ions adsorption onto the magnesium loaded activated carbons progressed via ion exchange mechanism. Based on the Boehm titration surface analysis, the strong heavy metal adsorption sites might be generated forming the magnesium bound to carbon surface via oxygen bridge by means of calcining the magnesium nitrate impregnated out-gassing carbon in air at $653 \mathrm{~K}$.
\end{abstract}

\section{KEYWORDS : Activated carbon, Calcination, Coating, Adsorption, Surface properties}

\section{Introduction}

Heavy metals are threat for human beings as well as ecosystems. A much amounts of heavy metals have been widely exhausted to the environment from the mining and industrial activities. Some of their ions are released to water environment such as acidic leachate from landfill ${ }^{1), 2)}$. Adsorptive removal of these contaminants is one of the attractive options. Numerous numbers of adsorbents have been applied to remove heavy metal ions form water environment such as agricultural waste, industrial by-products, zeolite, clay and activated carbon $\left.{ }^{3)}, 4\right)$. In general, carbonaceous materials including activated carbon exhibit excellent performance for adsorption of organic compounds, but they do not accommodate sufficient amount of heavy metal ions ${ }^{5)}$. In case of using carbons as heavy metals adsorbents, carbon surface oxidation by air, ozone, hydrogen peroxide and nitric acid can be generally employed as effective methods to increase the amount of heavy metal adsorption at the expense of decrease in adsorption for organic compounds, because carboxyls and lactones introduced to carbon surface by oxidation can be effective adsorption sites for heavy metal ions, but the $\mathrm{C}-\mathrm{O}$ complexes on the carbon reduce the $\pi$-electron density leading to the significant reduction of organic compounds adsorption ${ }^{5)}$. In contrast, complete removal of the $\mathrm{C}-\mathrm{O}$ complexes from the carbon surface by out-gassing at $1173 \mathrm{~K}$ also results in the increase of adsorption capacities of heavy metal ions caused by $\mathrm{C} \pi$-cation interaction $^{6)}$, and those of organic compounds will be enhanced though energy cost is increased by heating. On the other hand, metal oxides containing in or on the carbon can also be strong adsorption sites for heavy metal ions $\left.{ }^{5)}, 7\right)$. Comparing to $\mathrm{C}-\mathrm{O}$ complexes such as carboxyls and lactones which are strong electron withdrawing functional groups, the metal oxides are expected to affect a slight or no influence on the adsorption capacity of organic compounds because of holding the $\pi$ electron density unchanged from the original graphene sheet. In our previous study, zinc oxides loaded activated carbon could enhance the lead(II) ion uptake by a factor of up to 4.5 , operating electron static interaction between $\mathrm{ZnO}$ and $\mathrm{Pb}$ (II) ions, without decline of the amount of an organic compound adsorption ${ }^{5), 8)}$. Stronger and greater adsorption sites for heavy metal ions will be required for activated carbon. Yantasee et al. examined the effect of carbon surface amine on adsorption of heavy metal ions, and found that introduction of $-\mathrm{NH}_{2}$ onto activated carbon improved $K_{\mathrm{d}}$, the distribution coefficient 
value, of copper(II) ions by 140 times greater than that measured on the unmodified activated carbon, whereas $K_{\mathrm{d}}$ was only 4 times

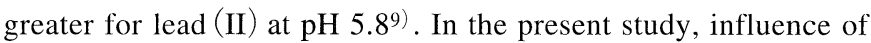
magnesium loading on lead(II) adsorption was inspected using activated carbon with various surface acidity. Meunier $e t$ al. pointed out that lead (II) uptake might be ion exchange with calcium, magnesium or potassium ions using high performance natural adsorbent of cocoa shells ${ }^{10)}$. Similar ion exchange mechanism with calcium and magnesium was proposed for other naturally obtained adsorbents such as brown seaweed ${ }^{11)}$ and olive stone waste $^{12)}$. In addition efficiency in removal of heavy metal ions of magnesium was considered to be preferable to calcium ${ }^{13)}$.

\section{Materials and methods}

\subsection{Adsorbents and their treatment and magnesium loading}

Adsorbent used was commercially available granular activated carbon (GAC), namely Diasorb W10-30 made from coconuts shell, purchased from Cargon Mitsubishi Corporation. At first, ash contained was completely eliminated from GAC by sequentially washing in hydrochloric acid and fluoric acid to get rid of the influence of mineral matters for the heavy metal adsorption. The acid treated GAC was repeatedly boiled in de-ionized water until the solution $\mathrm{pH}$ at ambient temperature was not changed any more. In the next step, the de-ashed GAC was oxidized to introduce acidic oxygen groups onto the GAC surface, or out-gassed to completely remove them from the surface. The oxygen functional groups were carboxylic, lactonic and hydroxyl groups. The oxidation was carried out warming the mixture of nitric acid and de-ashed GAC controlling the temperature at $363 \sim 368 \mathrm{~K}$. The oxidized GAC was rinsed for several times until a constant $\mathrm{pH}$ value was obtained, and then calcined at $653 \mathrm{~K}$ in air to decompose the nitric acid remaining in the carbon for 4 hours, and allowed to cool at room temperature in a desiccator. The amounts of the oxygen functional groups were altered changing the concentration of nitric acid and the warming time; for instance, GAC-Ox6.8-5 indicated that de-ashed GAC was oxidized with $6.8 \mathrm{M}$ nitric acid for 5 hours. The out-gassing to thoroughly remove the acidic groups was done at $1173 \mathrm{~K}$ in helium flow. The de-ashed GAC was placed in a quartz tube, and high purity helium gas was slowly introduced to the quartz tube to prevent the carbon from scattering toward the down flow direction. When the most of the inner tube volume was sufficiently replaced by helium gas, the tube temperature was increased to $383 \mathrm{~K}$ for 10 minutes and kept for 5 minutes in an electric furnace to remove the extra air and moisture remaining inside the activated carbon particle, and then the furnace temperature was raised up to the outgassing temperature up to $1173 \mathrm{~K}$ holding the temperature for 2 hours. In the final step, the quartz tube was removed from the furnace and cooled down to room temperature, kept helium flow for 5 minutes before contact with air, and finally it was stored in a desiccator. The out-gassed carbons were donated as GAC-OG.

Five hundred $\mathrm{mg}-\mathrm{Mg} / \mathrm{L}$ of magnesium(II) nitrate solution was prepared, and $100 \mathrm{~mL}$ solution and $1.5 \mathrm{~g}$ of the de-ashed activated carbon of GAC, GAC-OG or GAC-OG-Ox of various acidic groups content was mixed in conical flask and agitated at $100 \mathrm{rpm}$ and $298 \mathrm{~K}$ for 7 days. All chemicals used in the experiments were regent grade. The magnesium(II) impregnated activated carbon was separated from the aqueous solution by vacuum filtration, rinsed with de-ionized water. After drying in air oven at $373 \mathrm{~K}$, the activated carbon was calcined at $653 \mathrm{~K}$ for 4 hours to decompose nitrate ions as well. Only the calcined magnesium loaded GAC-OG was further out-gassed in helium flow at $1173 \mathrm{~K}$ to remove extra acidic functional groups formed by the calcination in air. The amount of magnesium loaded onto the activated carbons was measured by atomic absorption spectroscopy (AAS, Rigaku novAA 300) after dissolving the magnesium from the carbon by $1 \mathrm{M} \mathrm{HCl}$.

\subsection{Properties of the prepared adsorbents}

The analytical characterization of the prepared activated carbon was made by $\mathrm{N}_{2}$ adsorption-desorption at $77 \mathrm{~K}$ (Beckman Coulter Model 3100) and Boehm acid and base titration ${ }^{14), 15)}$. The BET surface area and pore size distribution were obtained from the $\mathrm{N}_{2}$ adsorption using the $t$-plots numerical analysis. Surface acidic functional groups and basic sites were determined using the Boehm titration. For the magnesium loaded GAC-OG, prior to the determination of acidic functional groups, it was washed with $\mathrm{HCl}$ solution of $\mathrm{pH} 2$, followed by out-gassed at $623 \mathrm{~K}$ in helium flow for 30 minutes.

A half gram of carbon and $15 \mathrm{~mL}$ of $0.1 \mathrm{M} \mathrm{NaHCO}_{3}, 0.05 \mathrm{M}$ $\mathrm{Na}_{2} \mathrm{CO}_{3}$ or $0.1 \mathrm{M} \mathrm{NaOH}$ solution was mixed in conical flask, and agitated at $100 \mathrm{rpm}$ for more than two days in room temperature. Five $\mathrm{mL}$ of the solution pipetted out for each sample was back titrated with $0.1 \mathrm{M} \mathrm{HCl}$. The $\mathrm{NaHCO}_{3}$ can neutralize only carboxylic groups on the carbon surface, $\mathrm{Na}_{2} \mathrm{CO}_{3}$ can react with both carboxylic and lactonic, and $\mathrm{NaOH}$ is able to react with all acidic groups of carboxyls, lactones and hydroxyls on the carbon. In accordance with the above assumption, the difference between the groups neutralized by $\mathrm{NaHCO}_{3}$ and $\mathrm{Na}_{2} \mathrm{CO}_{3}$ becomes lactones, the difference between those neutralized by $\mathrm{Na}_{2} \mathrm{CO}_{3}$ and $\mathrm{NaOH}$ is hydroxyls. The same procedure was carried out for the mixture of $0.5 \mathrm{~g}$ of the carbons and $15 \mathrm{~mL}$ of $0.1 \mathrm{M} \mathrm{HCl}$ solution to determine the basic sites of the carbon surface. The remaining $\mathrm{HCl}$ solution was titrated with $0.1 \mathrm{M} \mathrm{NaOH}$ after neutralization. Neutralization points were monitored using methyl red solution indicator for weak base titrated with strong acid, and phenolphthalein solution for strong acid and strong base combination.

\subsection{Adsorption measurements}

Lead (II) ions were used as a heavy metal dissolving lead(II) 
chloride to de-ionized water to adjust the concentration at $4.83 \mathrm{mmol} / \mathrm{L}$ for stock solution. The stock solution was diluted to desired concentration with de-ionized water. A hundred mg of the adsorbents was dosed to $50 \mathrm{~mL}$ of the aqueous solution, and agitated by $100 \mathrm{rpm}$ for more than 5 days at $293 \mathrm{~K}$ for the equilibrium experiments. The solution $\mathrm{pH}$ was measured by a portable $\mathrm{pH}$ meter (HORIBA Model D-51) for the initial and the equilibrium solutions. Metal ions concentration of lead (II) and magnesium(II) in the solution was determined using atomic absorption spectroscopy (AAS) as described in the previous section. Prior to the AAS analysis, the solution was diluted with de-ionized water and a drop of $1 \mathrm{M} \mathrm{HCl}$ to the external standard concentration range. The $\mathrm{HCl}$ acid was added to convert all chemical species in the solution to $\mathrm{Pb}^{2+}$ or $\mathrm{Mg}^{2+}$ during the analysis for heavy metal ions.

Adsorption of an organic compound as well as heavy metal ions adsorption was also examined to compare the difference between with and without magnesium loading. Nitrobenzene was used as an organic compound because it was proven that its adsorption from aqueous solution was greatly influenced by surface nature and textural properties of activated carbons in our previous study ${ }^{16)}$.

\section{Results and discussion}

\subsection{Properties of adsorbents}

Total BET surface area, and those of meso- and macro-pore and micro-pore of de-ashed activated carbon, GAC, oxidized and outgassed GAC's, and their magnesium modified counterparts were also tabulated in Table 1. Since the extent of oxidation was varied, one example of $7.5 \mathrm{M}$ nitric acid oxidation for 8 hours is represented in the table as an oxidized activated carbon. As expected in the oxidation study, total BET surface area is clearly decreased by the introduction of $\mathrm{C}-\mathrm{O}$ complexes to the edge of graphene sheet widening most of micro-pore into meso- and macro-pore ${ }^{5)}$. Though there is no significant difference between GAC and magnesium loaded GAC, a little activation effect of magnesium loading is observed in increasing micro-pore surface area for the out-gassed GAC. Table 2 shows acidic functional groups and basic sites on

Table 1 BET specific surface area and pore distribution of adsorbents.

\begin{tabular}{lccc}
\hline \multicolumn{1}{c}{ Adsorbent } & $\begin{array}{c}\text { BET specific } \\
\text { surface } \mathrm{area}, \mathrm{m}^{2} / \mathrm{g}\end{array}$ & $\begin{array}{c}\text { Meso-\&Macro- } \\
\text { pore } \mathrm{area}, \mathrm{m}^{2} / \mathrm{g}\end{array}$ & $\begin{array}{c}\text { Micro-pore } \\
\text { area, } \mathrm{m}^{2} / \mathrm{g}\end{array}$ \\
\hline GAC & 1120 & 120 & 1000 \\
GAC-MgO & 1120 & 130 & 990 \\
GAC-Ox7.5-8 & 840 & 680 & 160 \\
GAC-OG & 1040 & 220 & 820 \\
GAC-OG-MgO-OG & 1190 & 150 & 1040 \\
\hline
\end{tabular}

GAC : de-ashed coconuts shell granular activated carbon GAC-MgO : magnesium loaded GAC

GAC-Ox7.5-8 : GAC oxidized with 7.5 $\mathrm{M} \mathrm{HNO}_{3}$ for 8 hours

GAC-OG : GAC out-gassed at $1173 \mathrm{~K}$

GAC-OG-MgO-OG : magnesium loaded GAC-OG followed by outgassing at $1173 \mathrm{~K}$ the activated carbon surface determined by Boehm titration. The more the $\mathrm{HNO}_{3}$ concentration and oxidation time, the more the acidic functional groups are formed on the carbon surface. On the other hand, almost all carboxylic and lactonic groups are eliminated from the carbon surface by out-gassing at $1173 \mathrm{~K}$, but some hydroxyl groups are remained on the surface.

\subsection{Amount of magnesium loaded on GAC}

As described in the previous section, the magnesium loaded to the activated carbons were prepared by calcining the magnesium(II)

Table 2 Surface acidic functional groups and basic sites by Boehm titration

\begin{tabular}{lcccc}
\hline \multicolumn{1}{c}{ Adsorbent } & $\begin{array}{c}\text { Carboxylic } \\
(-\mathrm{COOH}) \\
\mathrm{mmol} / \mathrm{g}\end{array}$ & $\begin{array}{c}\text { Lactonic } \\
(-\mathrm{COO}-) \\
\mathrm{mmol} / \mathrm{g}\end{array}$ & $\begin{array}{c}\text { Hydroxyl } \\
(-\mathrm{OH}), \\
\mathrm{mmol} / \mathrm{g}\end{array}$ & $\begin{array}{c}\text { Basic sites } \\
(\mathrm{C} \pi), \\
\mathrm{mmol} / \mathrm{g}\end{array}$ \\
\hline GAC & 0.03 & 0.04 & 0.15 & 0.47 \\
GAC-OG & 0.00 & 0.00 & 0.08 & 1.10 \\
GAC-OG-MgO-OG-AT & 0.00 & 0.01 & 0.38 & - \\
GAC-OG-Ox0.85-0.25 & 0.39 & 0.36 & 0.65 & 0.10 \\
GAC-OG-Ox0.85-0.5 & 0.42 & 0.36 & 0.60 & 0.17 \\
GAC-OG-Ox0.85-1 & 0.45 & 0.17 & 0.61 & 0.14 \\
GAC-OG-Ox0.85-5 & 0.74 & 0.26 & 0.41 & 0.04 \\
GAC-OG-Ox1.7-5 & 0.96 & 0.22 & 0.29 & 0.04 \\
GAC-OG-Ox3.4-1 & 0.53 & 0.15 & 0.38 & 0.14 \\
GAC-OG-Ox3.4-2.5 & 0.74 & 0.28 & 0.42 & 0.06 \\
GAC-OG-Ox3.4-5 & 1.10 & 0.29 & 0.24 & 0.00 \\
GAC-OG-Ox6.8-5 & 0.91 & - & - & - \\
GAC-OG-Ox13.6-5 & 1.20 & - & - & - \\
\hline
\end{tabular}

GAC : de-ashed coconuts shell granular activated carbon GAC-Ox3.4-5: GAC oxidized with $3.4 \mathrm{M} \mathrm{HNO}_{3}$ for 5 hours GAC-OG : GAC out-gassed at $1173 \mathrm{~K}$

GAC-OG-MgO-OG-AT : magnesium loaded GAC-OG followed by outgassing at $1173 \mathrm{~K}$, and then washed with $\mathrm{pH} 2$ acid solution

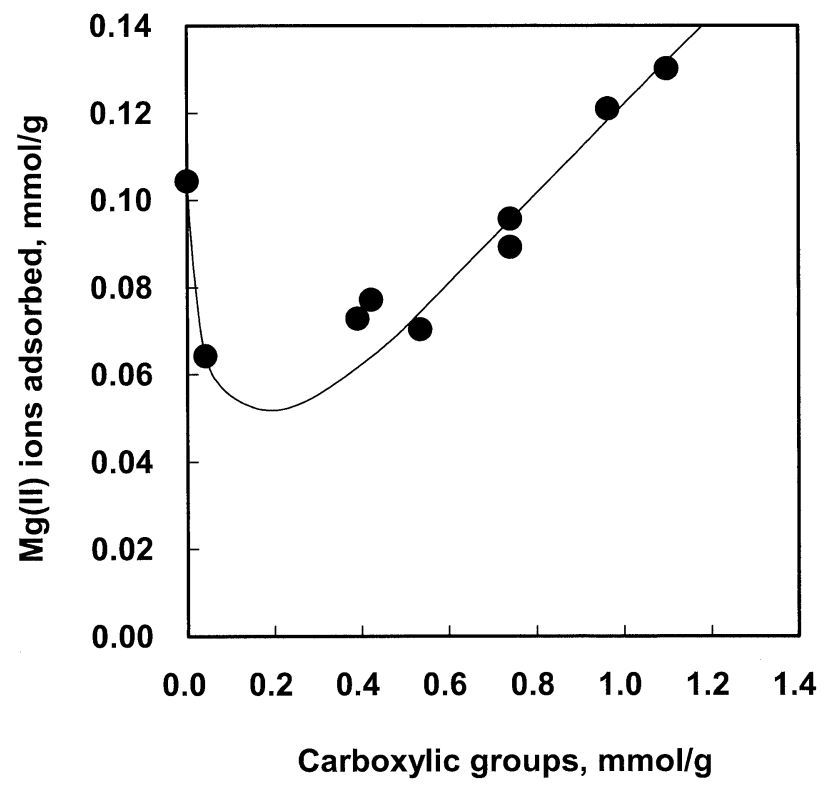

Fig.1 Changes in the adsorption amount of magnesium(II) ions onto the de-ashed coconuts shell based activated carbons in aqueous solution as a function of the carboxyls amount on the carbon surface determined by Boehm titration. 
ions pre-adsorbed carbons at $653 \mathrm{~K}$. The amounts of loaded magnesium were depicted in Fig.1 as a function of carboxyl groups, because the adsorption of divalent $\mathrm{Mg}^{2+}$ is principally considered to proceed via ion exchange with two carboxylic protons. The minimum point in Fig.1 was observed at the carboxyls amount of around $0.2 \mathrm{mmol} / \mathrm{g}$. Though the amount of magnesium is proportional to the carboxyl groups content above $0.2 \mathrm{mmol} / \mathrm{g}$ carboxyls, it is also increased by the very small amount of carboxyls below $0.2 \mathrm{mmol} / \mathrm{g}$. Only a small amount of carboxylic is known to become sufficient amount for diminishing $\pi$ electron density on the graphene sheet ${ }^{6)}$. Once the $\mathrm{C}-\mathrm{O}$ complexes are thoroughly removed, the withdrawal $\pi$ electron comes back to the graphite sheet, then interaction between $\pi$ electron and magnesium(II) ions, namely $\mathrm{C} \pi$-cation interaction, is working instead of ion exchange process valid in the presence of carboxyls. The sharp increase in magnesium adsorption at the carboxyls amount approaching zero may be attributed to the increase in $\pi$ electron density on the graphene sheet, since amount of basic sites reflecting $\mathrm{C} \pi$ density is also increased by out-gassing as can be clearly seen in Table $2^{6)}$.

\subsection{Influence of magnesium loading on $\mathrm{Pb}$ (II) adsorption}

Influence of magnesium loading to activated carbon on $\mathrm{Pb}$ (II) ions adsorption was examined using de-ashed carbon of GAC, oxidized of GAC-Ox and out-gassed of GAC-OG. The solution $\mathrm{pH}$ was always less than 6.5 throughout the experiments, and most of the $\mathrm{Pb}(\mathrm{II})$ aqueous species in that $\mathrm{pH}$ range could be $\mathrm{Pb}^{2+}$, but may be partly present as $\mathrm{Pb}(\mathrm{OH})+$ by the maximum $10 \%$ at the

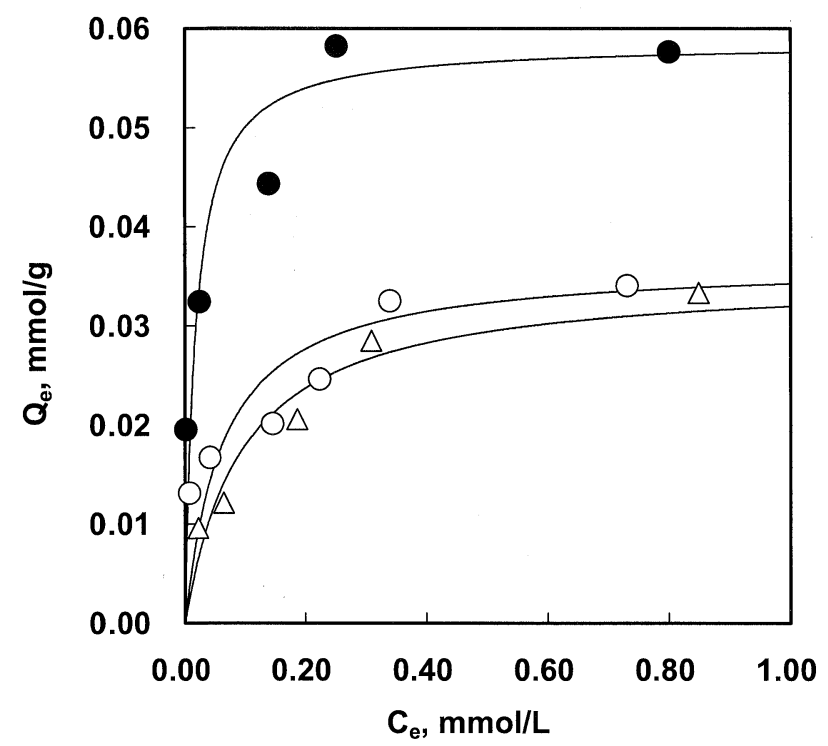

Fig.2 $\mathrm{Pb}$ (II) ions adsorption isotherms at $293 \mathrm{~K}$ before and after magnesium loading to the de-ashed activated carbon. $\bigcirc$; de-ashed activated carbon (GAC), ; magnesium loaded GAC (GAC-MgO), $\triangle ; 1 \mathrm{M} \mathrm{HCl}$ washed GAC-MgO. Solid lines : predicted using Langmuir parameters obtained by experimental results.
pH approaching 6.5. As shown in Fig.2, magnesium loading of GAC-MgO enhanced the $\mathrm{Pb}$ (II) adsorption obeying Langmuir isotherm as well as that without magnesium. The adsorption isotherm was come back to the original isotherm of GAC by washing GAC-MgO with $1 \mathrm{M} \mathrm{HCl}$ to remove loaded magnesium, revealing that the magnesium played an important role in $\mathrm{Pb}$ (II) adsorption. Fig.3 represents the effect of magnesium loading onto oxidized carbon of GAC-Ox containing carboxylic groups about $1 \mathrm{mmol} / \mathrm{g}$. As can be clearly seen, no enhancement of $\mathrm{Pb}$ (II) species was obtained by magnesium loading on GAC-Ox. The results suggested that the magnesium loading onto GAC-Ox and consecutive calcination, denoted to GAC-Ox-MgO, cannot change the magnesium chemical species, probably revealing that only ion exchanged $\mathrm{Mg}^{2+}$ species with carboxyl protons was formed on the carbon surface. In case of the magnesium loading to GAC-OG which was out-gassed at $1173 \mathrm{~K}$ in the final stage to remove acidic groups formed by the calcination in air at $653 \mathrm{~K}$ after magnesium loading, referred to as GAC-OG-MgO-OG, considerable enhancement of $\mathrm{Pb}$ (II) adsorption could be measured against the counterpart before magnesium loading of GAC-OG as drawn in Fig.4. The absolute amount of $\mathrm{Pb}$ (II) onto GAC-Ox indeed is greater than those onto GAC-OG irrespective of magnesium loading or not, but considerable reduction in adsorption amounts of organic compounds is necessarily observed for the oxidized GAC comparing to the non-oxidized carbon. Before the adsorption experiments, it was confirmed that no promoting effect could be observed for the $\mathrm{Pb}(\mathrm{II})$ adsorption onto the magnesium loading out-gassed GAC without calcination at

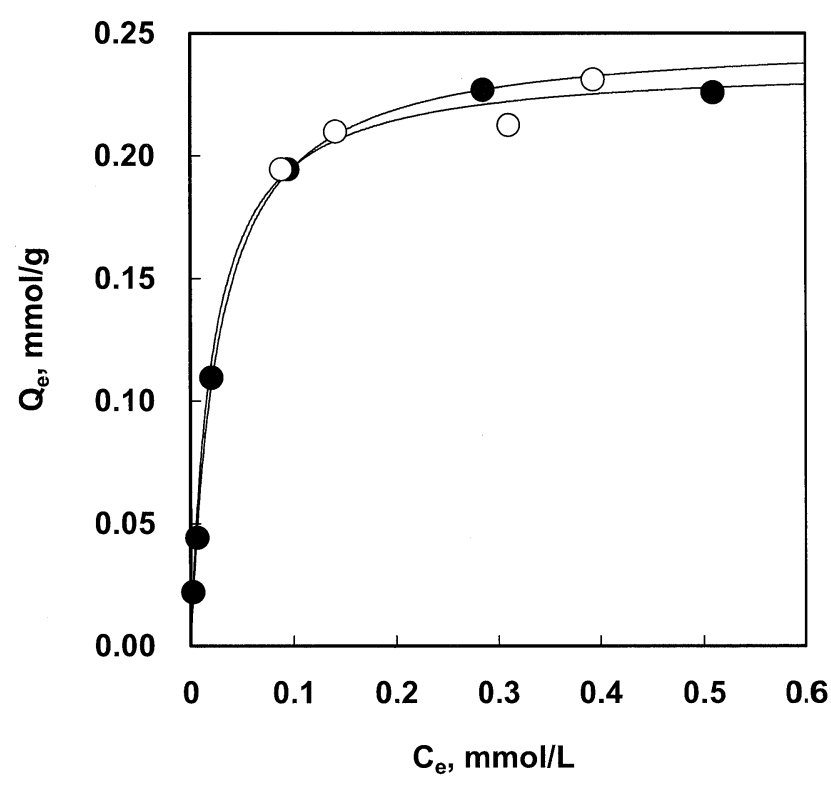

Fig.3 $\mathrm{Pb}$ (II) ions adsorption isotherms at $293 \mathrm{~K}$ before and after magnesium loading to the activated carbon oxidized with nitric acid. $\bigcirc$; oxidized activated carbon (GAC-Ox), $\mathrm{O}$; magnesium loaded GAC-Ox (GAC-Ox-MgO). Solid lines : predicted using Langmuir parameters. 
$653 \mathrm{~K}$, suggesting that the magnesium species would be changed from $\mathrm{C} \pi$-cation interaction to chemical binding to the carbon by the calcination. As drawn by the solid lines in Figs. 2 through $\mathbf{4}$, the experimental results can be predicted by Langmuir isotherms as indicated blow,

$$
Q_{e}=\frac{K_{e} C_{e}}{1+K_{e} C_{e}} X_{m}
$$

where $Q_{e}$ is the equilibrium amount of $\mathrm{Pb}$ (II) on the adsorbent, $C_{e}$ is the equilibrium $\mathrm{Pb}(\mathrm{II})$ solution concentration, and $K_{e}$ and $X_{m}$ represent the Langmuir adsorption affinity and capacity, respectively. The $K_{e}$ and $X_{m}$ values calculated from the experimental plots are tabulated in Table 3 for GAC, GAC-Ox and GAC-OG with and without magnesium loading. Though the adsorption capacities of $X_{m}$ 's were not altered to much extent for all adsorbents by loading magnesium, the adsorption affinities of $K_{e}{ }^{\prime}$ s were increased for GAC and GAC-OG, revealing that not the number but the quality of adsorption sites might be changed by magnesium loading. Particularly, the $K_{e}$ value for GAC-OG-MgO-OG was

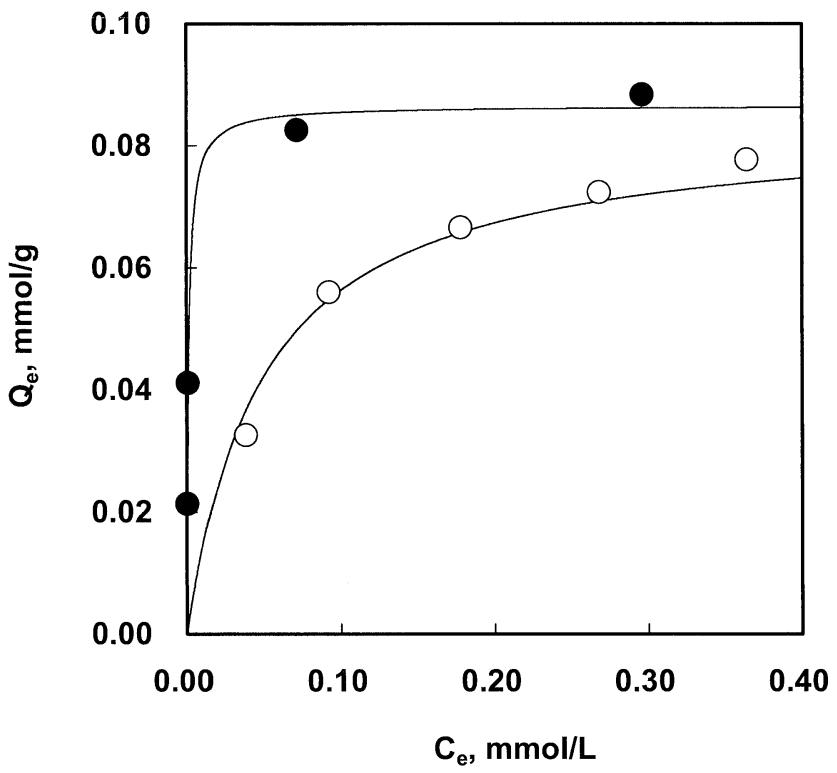

Fig.4 $\mathrm{Pb}$ (II) ions adsorption isotherms at $293 \mathrm{~K}$ before and after magnesium loading to the activated carbon out-gassed in helium at $1173 \mathrm{~K}$. $\bigcirc$; out-gassed activated carbon (GACOG), ; magnesium loaded GAC-OG (GAC-OG-MgO) . Solid lines : predicted using Langmuir parameters.

Table 3 Langmuir parameters for $\mathrm{Pb}$ (II) adsorption in aqueous solution

\begin{tabular}{ccc}
\hline \multicolumn{1}{c}{ Adsorbent } & $X_{m}, \mathrm{mmol} / \mathrm{g}$ & $K_{e}, \mathrm{~L} / \mathrm{mmol}$ \\
\hline GAC & 0.04 & 16 \\
GAC-MgO & 0.06 & 59 \\
\hline GAC-Ox & 0.24 & 47 \\
GAC-Ox-MgO & 0.25 & 37 \\
\hline GAC-OG & 0.08 & 20 \\
GAC-OG-MgO-OG & 0.09 & 450 \\
\hline
\end{tabular}

more than 20 times as great as that for GAC-OG. Fig.5 shows the plots of the amount of magnesium(II) ions desorbed from the GAC-OG-MgO-OG against those of $\mathrm{Pb}$ (II) ions adsorbed onto the carbon. It can be suggested that the $\mathrm{Pb}$ (II) adsorption proceeds via ion exchange with magnesium(II) ions from the results, through the approximate line in Fig.5 exhibits an intercept in the vertical axis revealing that a part of magnesium ions having no interaction to the carbon surface are released without the ion exchange with $\mathrm{Pb}$ (II) ions, probably due to magnesium remained without adsorption and/or separate from the surface in the GAC pore in the preparation procedure. When the GAC-OG-MgO-OG was washed with $\mathrm{pH} 2$ nitric acid to remove the ion exchangeable magnesium(II), the remaining acidic sites are only hydroxyl groups as also represented in Table 2 by GAC-OG-MgO-OG-AT, and the amount of phenolic groups was increased by $0.30 \mathrm{mmol} / \mathrm{g}$ comparing to $\mathrm{GAC}-\mathrm{OG}$, and the $0.30 \mathrm{mmol} / \mathrm{g}$ was more than equivalent amount of $0.2 \mathrm{mmol} / \mathrm{g}$ of $0.1 \mathrm{mmol} / \mathrm{g}$ magnesium(II) loading as seen in Fig.1. From these experimental results, the strong $\mathrm{Pb}(\mathrm{II})$ adsorption sites represented by $K_{e}$ value $450 \mathrm{~L} / \mathrm{mmol}$ can be supposed to be magnesium(II) bound to the edge of graphite carbon via oxygen bridge denoted to as $\mathrm{C}-\mathrm{O}-\mathrm{Mg}-\mathrm{O}-\mathrm{C}$, in which " $\mathrm{C}$ " is represented as graphite carbon, though the hydroxyl groups by themselves cannot be effective.

For the de-ashed activated carbon of GAC with magnesium loading, the adsorption affinity of $\mathrm{Pb}$ (II) was more than five times greater than without magnesium. In our preliminary study, adsorption sites were considered to be able to be divided into two types for GAC; one is the acidic sites of carboxylic and lactonic groups, the other is

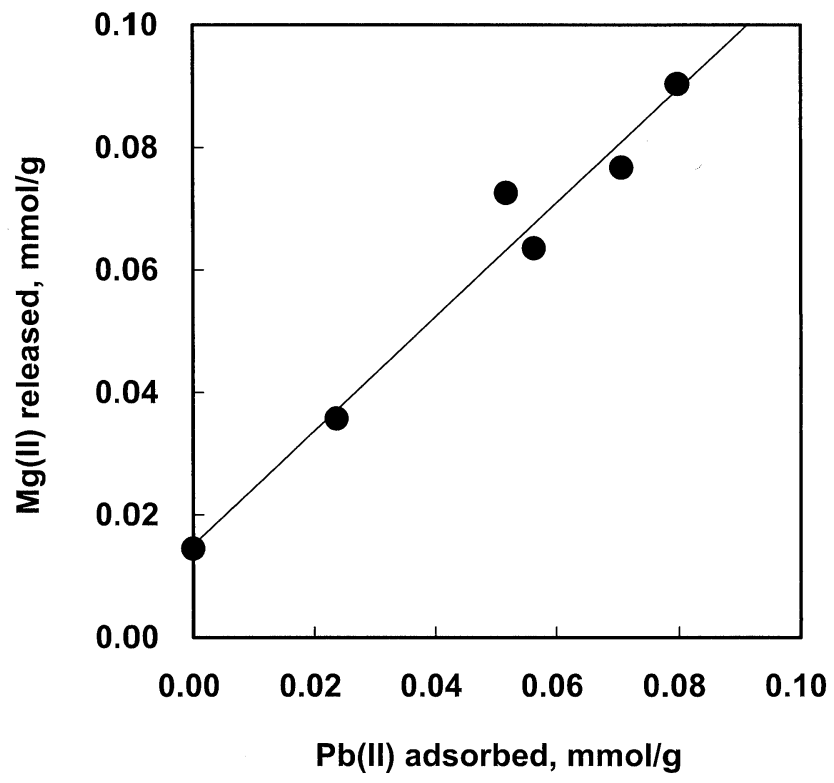

Fig.5 Relationship between $\mathrm{Mg}$ (II) ions released from magnesium loaded activated carbon (GAC-OG-MgO-OG) and $\mathrm{Pb}$ (II) adsorbed onto the carbon obtained by the adsorption kinetics experiments. 


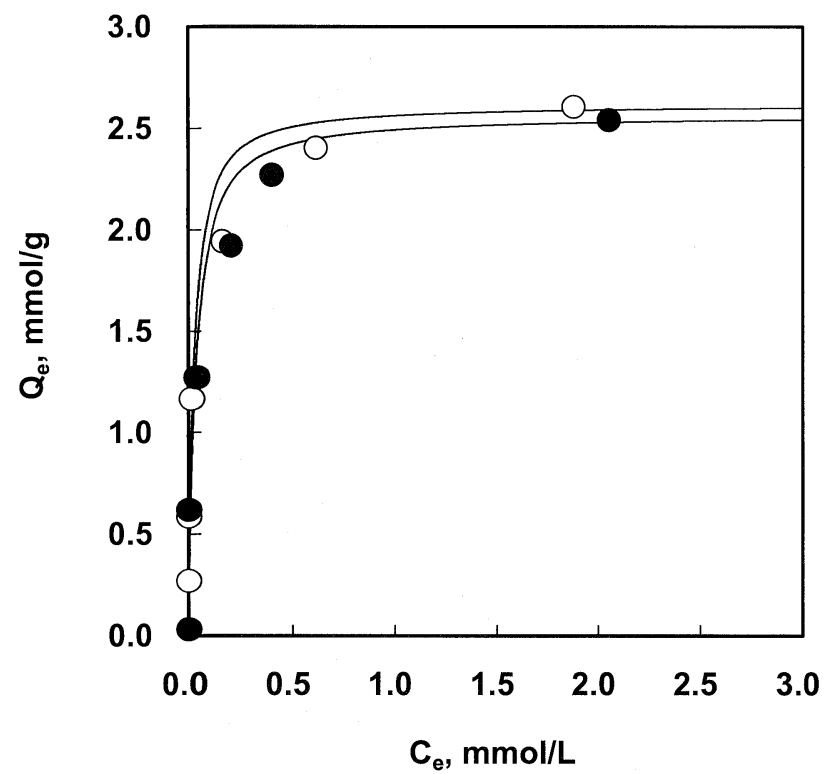

Fig.6 Nitrobenzene adsorption isotherms at $293 \mathrm{~K}$ before and after magnesium loading to the activated carbon out-gassed in helium at $1173 \mathrm{~K}$. $\bigcirc$; out-gassed activated carbon (GACOG), ; magnesium loaded GAC-OG (GAC-OG-MgO) . Solid lines : predicted using Langmuir parameters.

Table 4 Langmuir parameters for nitrobenzene adsorption in aqueous solution

\begin{tabular}{lcc}
\hline \multicolumn{1}{c}{ Adsorbent } & $X_{m}, \mathrm{mmol} / \mathrm{g}$ & $K_{e}, \mathrm{~L} / \mathrm{mmol}$ \\
\hline GAC-OG & 2.8 & 20 \\
GAC-OG-MgO-OG & 2.7 & 22 \\
\hline
\end{tabular}

basic sites caused by $\mathrm{C} \pi$ electron on the graphene layer ${ }^{6)}{ }^{17)}$. The adsorption affinity of $\mathrm{Pb}$ (II) onto GAC can be the average value of the acidic and basic sites. Based on the results on Table 3 , magnesium ions adsorbed on basic sites of GAC are estimated to be converted from the $\mathrm{C} \pi$-cation to the stronger ion exchangeable $\mathrm{C}-\mathrm{O}-\mathrm{Mg}-\mathrm{O}-\mathrm{C}$ sites on the surface by the calcination, whereas those on acidic sites are no longer changed. The $K_{e}$ value of $59 \mathrm{~L} / \mathrm{mmol}$ might be, therefore, reflected by both acidic sites and C-O-Mg-O-C sites. The acidic sites are estimated to be principally working for $\mathrm{Pb}$ (II) ions adsorption on oxidized GAC-Ox-MgO, while only the newly introduced C-O-Mg-O-C strong sites can be operative for out-gassed GAC-OG-MgO-OG.

\subsection{Influence of magnesium loading on nitrobenzene adsorption}

Influence of magnesium(II) loading to activated carbon of GAC-OG on the adsorption of nitrobenzene was shown in Fig.6 and Table 4. No significant difference between with and without magnesium was thankfully observed, even though strong adsorption sites for $\mathrm{Pb}$ (II) ions was developed on the carbon surface. In contrast to the magnesium loading, oxidation of activated carbon considerably reduced the adsorption capacity of aromatics forming electron withdrawing functional groups of carboxyls and lactones on the carbon surface. It is generally accepted that the aromatic compounds adsorb on the non-oxidized graphitic part of the surface physically through dispersive interactions between the $\pi$ electrons of the graphitic carbon basal planes and those of the aromatic ring, namely $\pi-\pi$ dispersive force $\left.{ }^{18)}-20\right)$. Decreasing $\pi$ electron density on the graphene sheet, therefore, directly lead to the reduction of aromatics adsorption ${ }^{21)}$. Consequently, magnesium loading affected no influence on $\pi$ electrons density of the carbon at all. Based on the experimental results of the adsorption of the heavy metal ions and aromatics, it can be concluded that the strong adsorption sites for $\mathrm{Pb}$ (II) adsorption are generated on the activated carbon by calcining $\mathrm{C} \pi$-magnesium(II) cation species at $653 \mathrm{~K}$ in air without any significant decline in adsorption capacity of the aromatic compound.

\section{Summary}

Influence of magnesium(II) loading to coconuts shell based activated carbons possessing various surface oxygen heterogeneity on the adsorption of $\mathrm{Pb}$ (II) ions and nitrobenzene in aqueous solution. The following conclusions might be deduced to summarize the experimental results.

1) The amounts of surface oxygen acidic groups can be controlled by changing nitric acid concentration and oxidation time.

2) Oxidation of the activated carbon increase the adsorption amount of magnesium(II) ions being proportional to carboxyl groups, but out-gassing also enhance the adsorption. The ion exchange with carboxyl protons can be working in the former, but the $\mathrm{C} \pi$-cation interaction shall be operative for the latter.

3) Introducing magnesium(II) ions onto the activated carbon by impregnation in aqueous solution followed by calcination at $653 \mathrm{~K}$, the Langmuir adsorption affinity of $\mathrm{Pb}$ (II) ions was enhanced more than 20 times as great as that without magnesium loading for the activated carbon out-gassed in helium at $1173 \mathrm{~K}$, whereas there was no effect of magnesium loading for the oxidized activated carbon.

4) $\mathrm{Pb}(\mathrm{II})$ adsorption for the magnesium loading out-gassed activated carbon would proceeded by ion exchange mechanism, because the equivalent amount of magnesium ions was released into aqueous solution in the process of $\mathrm{Pb}$ (II) ions adsorption.

5) The strong adsorption sites for $\mathrm{Pb}$ (II) ions were estimated to be made up of magnesium(II) binding to graphite carbon via oxygen bridge since only hydroxyl groups can be detected by Boehm titration after acid washing the magnesium loaded carbon.

6) No influence of magnesium loading on adsorption of organic compounds might be expected, because the adsorption isotherms of nitrobenzene were not varied at all by the introduction of magnesium onto out-gassed activated carbon. 


\section{Acknowledgements}

The authors thank Dr. Keiichi Nagao, the head of Safety and Health Organization in Chiba University, for his financial support and great encouragement on our works.

\section{References}

1) J. J. Dijkstra, J. C. L. Meeussen and R. N. J. Comans, Environ. Sci. Technol. 38 (2004) 4390-4395.

2) Y. -C. Jang and T. G. Townsend, Environ. Sci. Technol. 37 (2003) 4778-4784.

3) T. A. Kurniawan, G. Y. S. Chan, W. Lo and S. Babel, Sci. Total Environ. 366 (2006) 409-426.

4) D. Mohan and C. U. Pittman Jr., J. Hazard. Mater. B137 (2006) 762-811.

5) Y. Kikuchi, Q. Qian, M. Machida and H. Tatsumoto, Carbon 44 (2006) 195-202.

6) M. Machida, T. Mochimaru and H. Tatsumoto, Carbon 44 (2006) 2681-2688.

7) M. Machida, R. Yamazaki, M. Aikawa and H. Tatsumoto, Sep. Purif. Technol. 46 (2005) 88-94.

8) C. Y. Yin, M. K. Aroua and W. M. A. W. Daud, Sep. Purif. Technol. 52 (2007) 403-415.
9) W. Yantasee, Y. Lin, G. E. Fryxell, K. L. Alford, B. J. Busche and C. D. Johnson, Ind. Eng. Chem. Res. 43 (2004) 2759-2764.

10) N. Meunier, J. Laroulandie, J. F. Blais and R. D. Tyagi, Bioresour. Technol. 90 (2003) 255-263.

11) R. Aravindhan, B. Madhan, J. R. Rao, B. U. Nair and T. Ramasami, Environ. Sci. Technol. 38 (2004) 300-306.

12) N. Foil, I. Villaescusa, M. Martinez, N. Miralles, J. Poch and J. Serarols, Sep. Purif. Technol. 50 (2006) 132-140.

13) I. L. Shashkova, A. I. Rat'ko and N. V. Kitikova, Colloids Surf., A 160 (1999) 207-215.

14) H. P. Boehm, Carbon 40 (2002) 145-149.

15) H. P. Boehm, Carbon 32 (1994) 759-769.

16) M. Machida, M. Hayashida, Y. Kato and H. Tatsumoto, TANSO 2006 [No.224] 266-271.

17) M. Machida, Y. Kikuchi, M. Aikawa and H. Tatsumoto, Colloids Surf., A 240 (2004) 179-186.

18) F. Villacañas, M. F. R. Pereira, J. J. M. Órfão and J. L. Figueiredo, J. Colloid Interface Sci. 293 (2006) 128-136.

19) L. R. Radvic, I. F. Silva, J. I. Ume, J. A. Menéndez, C. A. Leon y Leon and A. W. Scaroni, Carbon 35 (1997) 1339-1348.

20) R. W. Coughlin and F. S. Ezra, Environ. Sci. Technol. 2 (1968) 291-297.

21) M. Franz, H. A. Arafat and N. G. Pinto, Carbon 38 (2000) 1807-1819. 